\title{
Care Diskurse und Praktiken ökologischer Foodways
}

Elisabeth Kosnik

In der Forschungsregion Oststeiermark erfolgte die weitgehende Umstellung der Landwirtschaft, weg von Selbstversorgung und hin zur Marktproduktion, teilweise erst in den 1970er Jahren und damit, im Vergleich $\mathrm{zu}$ anderen Regionen Westeuropas, verhältnismäßig spät. ${ }^{1}$ Historiker*innen betrachten diesen tiefgreifenden und rasanten Wandlungsprozess in der Region unterschiedlich, von positiv bis kritisch. ${ }^{2}$ Etwa zur selben Zeit begann sich die ökologische Produktionsweise in Österreich zu verbreiten. ${ }^{3}$ Dass diese Form der Landwirtschaft aus einer Kritik an der industriellen, chemisch-technischen Landwirtschaft hervorging, ist eine vielfach wiederholte Aussage und gleichzeitig eine vereinfachende Perspektive, die zumal vorrangig die Sichtweise der Konsument*innen wiedergibt. Die Motive für eine Umstellung auf ökologische Produktion waren und sind vielfältig, wie im Folgenden aufgezeigt wird.

Dieses Kapitel befasst sich mit den historischen und gesellschaftlichen Kontexten, in welche die Fallbeispiele unserer Studie eingebettet sind. Dabei soll insbesondere auf Diskurse und Entwicklungen im

\footnotetext{
1 Vgl. Eberhart 1991.
}

2 Siehe hierzu die eher positive Darstellung bei Posch 1978a, 1978b, bzw. die kritische Einschätzung von Kaser u.a. 2003, Kaser und Stocker 1986, 1987.

Die Bezeichnung »ökologischer Landbau« dient hier als Überbegriff für sämtliche ökologische Landbausysteme und in Abgrenzung zum sogenannten »konventionellen « Landbau, beziehungsweise zu tradierten Formen von Landwirtschaft. Der in Österreich üblichere Begriff »bio« ist als Synonym zu verstehen. 
ökologischen Landbau im deutschsprachigen Raum eingegangen werden, beziehungsweise auf die Geschichte der Landwirtschaft der Forschungsregion Oststeiermark. Die spärliche Datenlage zu den Anfängen des ökologischen Landbaus in der Steiermark lässt dabei kaum Generalisierungen über Akteur*innen und ihre Motive $\mathrm{zu} .^{4}$ Gleichzeitig würde eine ausführliche Darlegung der historischen Entwicklungen der Landwirtschaft den Rahmen dieses Kapitels überschreiten. Der Fokus wird daher auf Aspekte gelegt, die im Kontext unserer Forschung besonders relevant erscheinen, wobei aus kulturwissenschaftlicher Perspektive die unterschiedlichen Diskursstränge mit den Mikroperspektiven der Akteur*innen, ihren Handlungen und Deutungshorizonten, zusammengedacht werden sollen.

Die Analyse baut auf den vier Fallbeispielen der vorliegenden Studie auf, erweitert durch meine seit 2009 laufenden ethnografischen Forschungen zu Leben und Arbeiten auf ökologischen Betrieben und Selbstversorgerhöfen. Im Zuge dieser Forschungen fanden mehrtägige Feldforschungsaufenthalte auf zwölf ökologischen Landwirtschaften im deutschsprachigen Raum statt, acht davon in der Steiermark. ${ }^{5}$ Es sind daher vorrangig die Produzent*innen, deren Praxen und Vorstellungen - in Bezug auf Produktion und Verarbeitung, Distribution und Konsum (kurz foodways) - die im Fokus dieses Kapitels stehen. Konkret geht es um Lebensmittelproduzent*innen, die Grundnahrungsmittel (Obst, Gemüse, Fleisch, Eier, Milch und Milchprodukte) für Menschen sich selbst und andere - produzieren.

Wie in der Einleitung zu diesem Band bereits angemerkt wurde, gibt es keinen politisch oder ideologisch homogenen ökologischen

4 Wichtige Hinweise zur Geschichte des ökologischen Landbaus in Österreich liefern die Recherchen von Aurelia Jurtschitsch 2010, 2009, 1998, sowie die Forschungsberichte der Bundesanstalt für Bergbauernfragen: Groier und Gleirscher 2005; Groier und Schermer 2005; Groier 2013. Zur Entwicklung des ökologischen Landbaus im deutschsprachigen Raum siehe: Vogt 2000, 2001a, 2001b; Willer und Schmid 2016; Staudenmaier 2013. Zur Reformbewegung in Österreich siehe z.B. Farkas 1998.

5 Vgl. Kosnik 2015, 2017, 2019. Weitere drei Höfe in Tirol (Österreich), einer in Thüringen (Deutschland). 
Landbau. Ökologischer Landbau war und ist mit unterschiedlichsten Weltanschauungen vereinbar, von Umweltschutz $\mathrm{zu}$ neoliberalem Marktrationalismus $\mathrm{zu}$ nationalistisch-völkischem Gedankengut. Trotz dieser Heterogenität von Akteur"innen, Motivationen und Praktiken lassen sich in der Geschichte des ökologischen Landbaus einige wesentliche Elemente erkennen, auf die im Folgenden genauer eingegangen wird. Dabei geht es nicht darum, vermeintliche Kontinuitäten aufzuzeigen. Vielmehr soll die Prozesshaftigkeit von Diskursen und Praktiken, insbesondere in Bezug auf das Konzept einer sorgsamen Landwirtschaft, betrachtet werden. María Puig de la Bellacasas Überlegungen $\mathrm{zu}$ soil care, human-soil relations und soil communities sollen hier insbesondere als theoretischer Rahmen fungieren. ${ }^{6}$ Als Forschungsfeld der "NaturenKulturen« (im Sinne einer Überwindung der Natur/Kultur-Dichotomie) werden dabei Beziehungen zwischen Menschen und anderen Lebewesen, beziehungsweise Ökosystemen (morethan-human) thematisiert und damit das bisherige Care-Verständnis feministischer Diskurse erweitert. ${ }^{7}$

Des Weiteren werden Jan Douwe van der Ploegs Begriffe des peasant farming und entrepreneurial farming ${ }^{8}$ als Denkfiguren herangezogen, um die differenzierten Entwicklungen im ökologischen Landbau zu analysieren (während die Kategorie capitalist/corporate farming - mobile AgroUnternehmen, die dort produzieren, wo Land und Arbeitskräfte am billigsten sind - hier weniger relevant erscheint). In Ploegs gewollt objektivierenden Kategorien steht peasant farming - unter anderem, aber insbesondere und im Gegensatz zu entrepreneurial farming - für Autonomiebestrebungen, etwa durch das Vermeiden von Schulden (Fremdkapital) und die Vermeidung von Verflechtungen mit vor- und nachgelagerten Industrien und damit den globalen Lebensmittelsystemen. »Thus, enlarging self-reliance and resilience are essential and this involves an enduring struggle for autonomy. «" Ploeg hebt dabei die Prozesshaftigkeit

\footnotetext{
6 Vgl. Puig de la Bellacasa 2014, 2015, 2019.

7 Vgl. Binder und Hess 2019.

8 Vgl. Ploeg 2010, 2014, 2018a, 2018b.

9 Ploeg 2018b, S. 30, Hervorhebung original.
} 
von peasantry hervor, als sich kontinuierlich (re-)konstituierend, transformierend, wachsend oder schwindend und sich dabei einer linearen Entwicklung hin zu entrepreneurial und capitalist farming verweigernd. ${ }^{10}$ Die Begriffe sind nicht streng voneinander trennbar, ihre Grenzen nach innen und außen verschwommen und auch in sich nicht als homogen $\mathrm{zu}$ verstehen. "Peasant farming contains a wide range of shades and nuances, which can range from pluriactivity to the situation of the landless and the many urban workers who cultivate plots for self-consumption ${ }^{11}{ }^{11}$ In diesem Kapitel sollen Ploegs Denkfiguren auf konkrete Fallbeispiele angewandt und erweitert werden.

Das Kapitel ist weitgehend chronologisch aufgebaut, beginnend mit den Anfängen institutionalisierter ökologischer Landwirtschaft in Österreich in der Nachkriegszeit - eine Zeitspanne, die sich mit den erinnerten Familienbiografien der Interviewten deckt. Im ersten Abschnitt wird die Rolle der Ernährungs- und Lebensreformbewegung für den ökologischen Landbau beleuchtet, die - insbesondere aus Eigensorge - zu einer ablehnenden Haltung ernährungsbewusster Konsument*innen und Produzent*innen gegenüber stickstoffhaltigem Mineraldünger und schwermetallhaltigen Pestiziden führte. ${ }^{12}$ CareDiskurse tauchen hier auch als »Versorgung des Bodens« auf, sowohl in der industrialisierten Landwirtschaft als auch im ökologischen Landbau. Diskurse der Ernährungsreform, so wird im zweiten Abschnitt deutlich, sind eng mit dem Vertrauensverlust in Produzent"innen verbunden. Konsument"innen forderten von der Landwirtschaft in ihrer Rolle als »Versorgerin der Gesellschaft« qualitätsvolle Nahrungsmittel und steigerten damit die Nachfrage nach anders produzierten Lebensmitteln. Der dritte Abschnitt beleuchtet die Entstehung einer "eco-entrepreneurial agriculture" (in Anlehnung an Ploegs Begriff) in den letzten drei Jahrzehnten, daher die Produktion ökologischer Waren für den Weltmarkt. Demgegenüber stehen aktuelle Konzepte von human-soil relations als sich gegenseitig versorgende Gemeinschaften

\footnotetext{
10 Vgl. Ploeg 2018b, S. 25, Ploeg 2018a.

11 Ploeg 2018b, S. 4.

12 Vgl. Vogt 2001b.
} 
von Mensch und Boden(-lebewesen), die abseits von Agrarromantik produktivistische Ideologien in Frage stellen.

\section{Die Rolle der Lebens- und Ernährungsreformbewegung für den ökologischen Landbau}

Nach dem zweiten Weltkrieg legte der sogenannte Marshall Plan ${ }^{13}$ die Richtung für die österreichische Wirtschaft fest. Die Landwirtschaft sollte modernisiert und für den westlichen Markt geöffnet werden. Staat und geldgebende Institutionen forcierten Wachstum und damit verbunden die chemisch-technische Intensivierung der landwirtschaftlichen Produktionsmethoden. Die "Produktionsgesinnung « ${ }^{14}$ wurde mithilfe staatlicher Berater*innen für landwirtschaftliche Betriebe, sowie von Landwirtschaftsschulen und -zeitschriften verbreitet - mit tatkräftiger Unterstützung der Kunstdüngerindustrie. ${ }^{15}$ Die Bodenwissenschaften der Zeit standen ebenfalls im Dienst dieser Entwicklung: Boden-Sorge wurde verstanden als Versorgung des Bodens mit $\mathrm{Zu}$ satzstoffen, um Pflanzenwachstum $\mathrm{zu}$ fördern und Schädlinge $\mathrm{zu}$ vernichten, wie Puig de la Bellacasa in ihrer Arbeit zu Bodenbeziehungen (human-soil relations) deutlich macht: "Soil care in a productionist frame is aimed at increasing soil's efficiency to produce for humans at the expense of all other relations «. ${ }^{16}$

Die Milchwirtschaft wurde in der zweiten Hälfte des 20. Jahrhunderts $\mathrm{zu}$ einem land- beziehungsweise marktwirtschaftlichen Schwerpunkt der Oststeiermark, sowie Massentierhaltung (insbesondere Schweine und Hühner) und Intensivobstbau. Ziegen, wie sie Familie Conrad heute hält, und Schafe gab es hingegen in der Region

13 US-amerikanisches Wiederaufbauprogramm für Europa (European Recovery Program, ERP).

$14 \quad$ Kaser und Stocker 1986, S. 197.

15 Vgl. Eberhart 1987; Jurtschitsch 2010; Kaser und Stocker 1987; Plakolm 2017.

16 Puig de la Bellacasa 2015, S. 700. 
nie in großer Zahl. ${ }^{17}$ Die Entwicklung der Landwirt*innen hin $\mathrm{zu}$ Rohstoffproduzent"innen, verflochten mit vor- und nachgelagerten Industrien (Ploegs entrepreneurial farming), lässt sich exemplarisch am Beispiel von Familie Bergmann nachzeichnen: Großvater Emil, selbst als staatlicher Landwirtschaftsberater tätig, stellte seinen Betrieb in der Nachkriegszeit früh auf Intensivobstbau um. In den 1970er Jahren folgte sein Sohn Richard neuen Empfehlungen zur stärkeren Spezialisierung und begann mit Geflügelwirtschaft. Küken und Futtermittel wurden aus dem Ausland bezogen, das Endprodukt (Bruteier) an fixe Abnehmer geliefert. Sein Sohn Martin bestätigt: »Es hat keinen fachlichen oder betrieblichen Grund gegeben, das zu ändern, nein, wir haben sicher nicht schlecht verdient in dieser Branche. Das war eine Nische und wenn du da gut warst, hast du gut damit verdient, keine Frage (Interview am 2.3.2018). Seit 2013 führt Martin Bergmann den Betrieb nun als ökologische Gemüse-CSA. Dabei wird die Familientradition, sich aktuellen Trends anzupassen, durchaus aufrechterhalten, wenn man die Entwicklungen und die fortschreitende gesellschaftliche Akzeptanz des ökologischen Landbaus in Österreich in Betracht zieht (siehe Kapitel Heistinger).

Allgemein führten die hohen Kosten für Investitionen seit der Nachkriegszeit zu einer breiten Verschuldungswelle unter den Landwirt*innen der Oststeiermark. Gleichzeitig blieben die positiven Effekte einer industrialisierten Landwirtschaft für die Region weit hinter den Erwartungen ihrer Befürworter*innen zurück. ${ }^{18}$ Die kleine Betriebsstruktur - typisch für das Flach- und Hügelland der Oststeiermark - erlaubte es den steirischen Landwirtschaftsbetrieben nie, im selben Ausmaß wie in anderen Regionen Europas in die Massenproduktion einzusteigen. ${ }^{19}$ Der Historiker Karl Stocker attestiert der Oststeiermark Mitte der 1980er Jahre eine »Anhäufung von wirtschaftlichen und sozialen Problemen, wie es sie kaum in einer anderen Region Österreichs in einer solchen Intensität gibt«. ${ }^{20}$

\footnotetext{
17 Vgl. Kaser und Stocker 1986; Posch 1978b.

18 Siehe dazu auch Ploeg 2010.

19 Vgl. Karner 2000.

20 Kaser und Stocker 1986, S. 267.
} 
Unterdessen gewann die Lebens- und Ernährungsreformbewegung in Österreich in den 1950er und 6oer Jahren verstärkt an Einfluss. Ernährungsreformerische Tendenzen gehen auf den im 19. Jahrhundert einsetzenden Ernährungswandel zurück, hin zu tierischen und verarbeiteten Nahrungsmitteln. »Das Nahrungsgefüge begann sich von einer wenig verarbeiteten, kohlenhydrat- und ballaststoffreichen hin $\mathrm{zu}$ einer fett- und eiweißreichen ballaststoffarmen Ernährung mit einem hohen Verarbeitungsgrad zu verschieben. ${ }^{21}$ Mitte des 19. Jahrhunderts begann sich die entstehende Lebensreformbewegung mit den möglichen negativen Folgen dieses Ernährungswandels auseinanderzusetzen. Über Vorträge, Vereine, Schriften und Schulungen - insbesondere durch den schwedischen Ernährungsreformer Are Waerland und das Schweizer Ehepaar Hans und Maria Müller - erreichten diese Ansätze die Steiermark. 1969 wurde der österreichische Demeter Bund (biologisch-dynamische Landwirtschaft basierend auf den anthroposophischen Grundsätzen Rudolf Steiners) gegründet. Darüber hinaus war es der seit 1963 jährlich erscheinende Mondkalender, den Maria Thun aus Hinweisen Steiners zur Aussaat entwickelt hatte, welcher weit über anthroposophische Kreise hinaus Verbreitung fand. So arbeitet auch Familie Bergmann in ihrer Gemüse-CSA nach dem Thunschen Aussaatkalender. ${ }^{22}$ Die Entwicklung ökologischer Anbausysteme lässt sich anhand der Geschichte ihrer Institutionen erkennen, allerdings sollte ihre Bedeutung nicht überbewertet werden. Es ist anzunehmen, dass Erfahrungen im ökologischen Landbau vielfach in undokumentierten Selbstversuchen gesammelt wurden, ohne engere Kontakte $\mathrm{zu}$ Institutionen und Netzwerken. ${ }^{23}$

Ernährungsbewusste, reformbewegte Verbraucher*innen betonten den in der Lebens- und Ernährungsreform wichtigen Zusammenhang von Produktionsmethoden, Nahrungsmittelqualität und Gesundheit. Ihre totale oder zumindest weitgehende Ablehnung von chemischen

21 Baumgartner 1998, S. 117.

22 Vgl. Vogt 2000, 2001b; Jurtschitsch 2010. Im Zuge meiner Forschungen ist mir diese Praxis vielfach begegnet; Kosnik 2019. 
Substanzen in der Nahrungsmittelerzeugung erhöhte die Nachfrage nach »naturbelassenen«, »ungespritzten« (daher ohne Chemie hergestellten) Lebensmitteln. ${ }^{24}$ Gleichzeitig spielten die vermehrt auf den Einsatz von Chemie zurückgeführten Krankheiten, Allergien und Spritzmittelvergiftungen in landwirtschaftlichen Familien, sowie die sich verschlechternde Futtermittelqualität und krankes Vieh, eine ausschlaggebende Rolle bei der Umstellung der Produktion auf ökologische Landbaumethoden. Ernährungsreform-orientierte Hausärzte, Tierärzte und Dorfpfarrer gaben oft die ersten Hinweise zu möglichen Alternativen. Insbesondere die Bäuerinnen fanden vielfach Interesse an einer Ernährungsumstellung, um die Gesundheit ihrer Familien zu fördern. Die Umstellung der Lebensmittelerzeugung erfolgte auf manchen Höfen als logische Konsequenz dieser Einstellungsänderung. ${ }^{25}$

Die Verbindung von Boden, Nahrung und Gesundheit hat sich als Themenkomplex im ökologischen Landbau erhalten. So formuliert der EU-Bericht zur ökologischen Landwirtschaft vom März 2019: ökologische Praktiken »address consumers demand for healthier food. The growing concern that excessive exposure to pesticides and overuse of antibiotics have a negative effect on health conditions is reorienting the society towards more natural alternatives. ${ }^{26}$

Dieselben Motive führen Interviewte in der vorliegenden Studie an, wobei Frauen zumindest teilweise nach wie vor die Rolle als Erhalterinnen der Gesundheit ihrer Familien übernehmen. Für Frau Adam etwa war Öko-Anbau von Anfang an eine Frage der gesunden Ernährung für sich und ihre Familie: "Ich esse gerne, ich koche gerne und das war die Ausgangsbasis für mich, mich selber gesund ernähren zu wollen« erklärt sie im Interview (20.8.2017). Frau Conrad bezeichnet die Nachfrage nach gesundheitsförderlicher magerer Milch als einen entscheidenden Faktor, Ziegenmilch entgegen den regionalen Normen zu produzieren. Außerdem geht sie gerne am Markt auf Fragen ihrer Kund"innen zu Gesund-

24 Vgl. Vogt 2000.

25 Vgl. Jurtschitsch 2010, 2009. Siehe die ähnliche Situation in Belgien: Vankeerberghen 2012.

26 European Commission 2019, S. 22. 
heit und Ernährung ein. Für die Familien Bergmann und Danninger hingegen waren es Krankheitsfälle in der Familie - Krebstod, chronische Erkrankungen und Vergiftungen durch Insektizide - welche einen wichtigen Einfluss auf die Entscheidung hatten, den Betrieb umzustellen. ${ }^{27}$

In Deutschland und Großbritannien entstand bereits um die letzte Jahrhundertwende eine Reformwarenwirtschaft, während Erfahrungen mit und Meinungen über ökologische Landbaumethoden durch zahlreiche Publikationen und Vorträge verbreitet wurden. ${ }^{28}$ In der Oststeiermark hingegen kam es, gleichzeitig mit anderen Regionen Österreichs, erst in den 1960er und 70er Jahren $\mathrm{zu}$ vermehrten Umstellungen auf alternative Landbaumethoden - zu einer Zeit, als Landwirt*innen bereits auf erprobtes Wissen und Erfolgsmodelle Anderer zugreifen konnten. Dabei dominierte in Österreich die sogenannte organisch-biologische Landbaumethode, wie die von Aurelia Jurtschitsch für Bio Austria durchgeführte Studie $\mathrm{zu}$ den »Bio Pionieren « Österreichs aufzeigt. ${ }^{29}$ Von der Ernährungsreformerin Maria Müller in Theorie und Praxis erarbeitet und von dem deutschen Arzt und Mikrobiologen Hans Peter Rusch wissenschaftlich fundiert, fand die Methode durch die Schulungs- und Vortragstätigkeiten Hans Müllers, sowie durch seine seit 1946 erscheinende Vierteljahresschrift Kultur und Politik weite Verbreitung. ${ }^{30}$

Ruschs Theorie der »lebendigen Substanz« propagierte ein alternatives Verständnis von Boden, das zwar weiterhin Bodenproduktivität zum Vorteil der Menschen in den Mittelpunkt stellte, dabei aber von einem komplexen Verständnis von Bodenbeziehungen ausging, welche

Eine wachsende Anzahl an Ethnografien beschäftigt sich mit den Zusammenhängen von Agrochemie und Gesundheitsschäden für Landwirt*innen, siehe Lock und Nguyen 2018.

28 In Deutschland z.B. Rudolf Steiners Landwirtschaftlicher Kurs (1924); in Großbritannien z.B. Sir Albert Howards The Influence of Soil Factors on Disease Resistance. Annals of Applied Biology, 1921, 7, S. 373-389.

29 Vgl. Jurtschitsch 2010; siehe auch Vogt 2000, 2001a, 2001b.

30 Vgl. Vogt 2000, 2001b; Wagner 2006; Lünzer 2002. 
durch Landwirtschaft nicht zerstört oder geschädigt werden sollen. Puig de la Bellacasa umschreibt dies mit dem Begriff der soil communities. ${ }^{31}$ Der Agrarsoziologe Gunter Vogt bezeichnet dieses »biologische Verständnis« von Bodenleben als einen Kernpunkt ökologischer Landbausysteme, ${ }^{32}$ das bis heute von großer Bedeutung ist, wie Wahlhütter u.a. in ihrer Studie zum Bodenverständnis von Landwirt"innen in Österreich feststellen. ${ }^{33}$ Puig de la Bellacasa unterstreicht die multi-species Dimension von soil life: »In this conception, soil is not just a habitat or medium for plants and organisms, nor is it just decomposed material, the organic and mineral end-product of organism activity. Organisms are soil. A lively soil can only exist with and through a multispecies community of biota that makes it«. 34

Die 1932 von Hans und Maria Müller in der Schweiz gegründete Landbau- und Volkshochschule wurde seit den 1960ern auch von zahlreichen österreichischen Landwirt*innen besucht, wobei Vogt den dort stattfindenden Wissensaustausch zwischen Praktiker*innen als wesentlich für die Weiterentwicklung des organisch-biologischen Landbaus betrachtet. ${ }^{35} 1960$ entstand in Österreich nach dem Vorbild von Hans Müllers Schweizerischer Bauern-Heimatbewegung die "Förderungsgemeinschaft für gesundes Bauerntum « als Interessensverband von Praktiker*innen und Theoretiker*innen. ${ }^{36}$ Laut Vogt fand in dieser Zeit ein grundlegender Wandel statt, von einer christlichkonservativen Ausrichtung hin zu einer ökologisch-sozialen: »Die bisherigen Grundsätze - Erhalt von Familie und Hof sowie Bewahrung von Heimat und Tradition - wurden durch Verantwortung für Natur und Verbraucherschaft ergänzt «. ${ }^{37}$

\footnotetext{
31 Vgl. Puig de la Bellacasa 2015, S. 704.

32 Vgl. Vogt 2001a.

33 Vgl. Wahlhütter u.a. 2016, S. 46.

34 Puig de la Bellacasa 2015, S. 701, Kursiv original.

35 Vgl. Jurtschitsch 2010, 1998; Vogt 2001b, 2000.

36 Vgl. Jurtschitsch 2010.

37 Vogt 2001b, S. 47-48.
} 


\section{Agrarkapitalismus und Landromantik - gegensätzliche Positionen im Agrardiskurs}

Seit den 1950er Jahren galt vielen Landwirt*innen der Oststeiermark Produktivismus als vielversprechendste Überlebensstrategie, wie Kaser und Stocker feststellen:

»Das wichtigste Gesetz dabei lautet, daß der Sinn der Produktion nicht mehr wie früher darin besteht, so viel am Hof zu produzieren, daß man davon leben kann, sondern möglichst billig zu produzieren, um die damit hergestellten Waren mit einem angemessenen Erfolg verkaufen zu können. Dabei muß egal sein, was man produziert, denn es geht in erster Linie darum, ein angemessenes Einkommen zu erwirtschaften. $\ll^{38}$

Demgegenüber stehen die in lebensreformerischen Ideen wurzelnden ökologischen Landbausysteme, denen eine moralische Ökonomie gemeinsam ist, welche Ideale wie Harmonie und Gemeinschaft einem individuellen Profitdenken vorziehen. So bezeichnet Hans Müller den gewinnorientierten Landwirt derogativ als »bäuerlichen Techniker «, ${ }^{39}$ im Gegensatz zum traditionell wirtschaftenden Bauern, den er als Dienenden beschreibt. In seiner Zeitschrift hält Müller fest: »Rechte Bauernarbeit ist immer dienen, ob sie der Dienstbote oder der freie Bauer tut. Dienst am Hofe - pflegen und hegen auf den Feldern oder in den Ställen ${ }^{40}{ }^{40}$ Laut Vogt leitet Müller Ideale wie Dienst und Verantwortung als bäuerliche Lebensmaximen aus seiner konservativ-christlichen Weltanschauung $a b$, »zum einen gegenüber der Familie als Lebensgemeinschaft und Tradition, zum anderen gegenüber der Natur als Heimat und Schöpfung ${ }^{41}$

38 Kaser und Stocker 1987, 228-229.

39 Kultur und Politik, Müller 1953, zitiert nach Vogt 2000, S. 197.

40 Kultur und Politik, Müller 1955, S. 27, zitiert nach Vogt 2000, S. 198.

41 Vogt 2000, S. 198. 
Agrarkapitalismus und Landromantik stehen sich als konträre politische Positionen im europäischen Agrardiskurs gegenüber, die weit in das 19. Jahrhundert zurückreichen. ${ }^{42}$ Ploeg stellt dazu fest:

»The problem with the historical debate is that it has frequently evolved into polarized and fixed positions that imbue the different sides of the equation with ontological properties. One camp has attributed peasant agriculture with an intrinsic superiority. The opposite position views peasant farming as intrinsically backwards. The dialectical relation between reality and potentiality (between > what-is<and > what-can-bes) has been neglected by both camps. ${ }^{43}$

Durch Bildungsarbeit der entsprechenden Institutionen (hier die Landwirtschaftskammer, dort lebensreformerische Volkshochschulen, beziehungsweise ihre Vereine und Zeitschriften) sollten die jeweiligen Standpunkte den Landwirt*innen näher gebracht werden. Tatsächlich existiert zwischen diesen Polen ein breites Spektrum an Diskursen und Praktiken realer Akteur*innen, die nicht auf Extreme verkürzt werden dürfen.

Während Staat und Wirtschaft nach der Grundmaxime agierten, dass nur eine industrialisierte Landwirtschaft die Bevölkerung ernähren könne, gingen die Anhänger*innen ökologischer Landbauweisen davon aus, dass der Anstieg an Quantität zwangsläufig mit dem Verlust von Qualität einhergehen müsse. In Anlehnung an E.P. Thompsons moral economy of provision ${ }^{44}$ ließe sich feststellen, dass für Befürworter*innen ökologischer Landbausysteme eine profitorientierte Landwirtschaft nicht länger ihre Aufgabe als Versorgerin der Bevölkerung mit nahrhaften Lebensmitteln erfüllen konnte.

Jurtschitsch zeigt auf, wie in den späten 1970er Jahren österreichische Printmedien und öffentliches Fernsehen vermehrt eine kritische Einstellung zur industrialisierten Landwirtschaft einnahmen. ${ }^{45}$ Es ent-

\footnotetext{
42 Vgl. Langthaler 2010.

43 Ploeg 2014, S. 1005.

44 Vgl. Thompson 1971.

45 Vgl. Jurtschitsch 2010.
} 
wickelte sich ein breiteres gesellschaftliches Bewusstsein für die Problematik von Rückständen chemisch-synthetischer Pestizide in Nahrungsmitteln. ${ }^{46}$ Mit Hilfe ökologischer Methoden sollte die Qualität der Erzeugnisse verbessert werden und die steigende Nachfrage den Produzent*innen gleichzeitig ein gutes Einkommen sichern. Während höhere Preise für Öko-Waren zu dieser Zeit noch kein Thema für die Produzent*innen waren, bedeutete der Verzicht auf zugekaufte Produktionsmittel eine deutliche Senkung der Produktionskosten. Nicht unbedeutend für die Verbreitung des Öko-Landbaus waren daher die Energiekrisen der 1970er Jahre, welche die Preise für chemisch erzeugten Stickstoffdünger steigen ließen und zahlreiche Landwirt*innen dazu veranlassten, eine weniger mechanisierte, Kunstdünger-freie Landwirtschaft in Betracht zu ziehen. ${ }^{47}$

Der gefühlte Vertrauensverlust - begründet darin, dass profitorientierte Unternehmen Quantität statt Qualität, beziehungsweise Profite auf Kosten von Gesundheit und Umwelt produzieren - setzt sich bis ins 21. Jahrhundert fort, wie Frau Adam im Interview beschreibt:

»Ich glaube, dass in den letzten Jahren einfach ein großer Vertrauensbruch stattgefunden hat, von Konsumentenseite, dass ich das Gefühl habe, es ist gerade voll viel Potential da für kleine Betriebe wie wir...es gibt schon einen starken Trend, finde ich, wo die Menschen qualitätsvoll sich ernähren wollen.« (Interview vom 20.8.2017)

Der bereits oben erwähnte EU-Bericht stellt ebenfalls fest: »The pursuance of quality food is a further driver. Organic food is sometimes associated with tastier and more nutrient-dense food, in line with the >flavour-over-appearance belief « ${ }^{48}$ Peasant farmers, ob ökologisch zertifiziert oder nicht, genießen in Österreich einen deutlichen Vertrauensvorschuss von Seiten der Konsument*innen, der von diesen Produzent"innen auch ökonomisch genutzt wird. So hat Frau Conrad die Erfahrung gemacht, dass nach Medienberichten über chemische Rück-

46 Vgl. Vogt 2000.

47 Vgl. Vogt 2000; Jurtschitsch 2010.

48 European Commission 2019, S. 8, eigene Hervorhebung. 
stände in Nahrungsmitteln die Nachfrage nach ökologischen Waren spürbar steigt, was auch der EU-Bericht bestätigt. ${ }^{49}$

\section{Öko-Entrepreneure und human-soil-relations}

Die Wachstums- und Intensivierungsbestrebungen im Landwirtschaftssektor führten seit den 1970ern $\mathrm{zu}$ einem Überschuss an landwirtschaftlichen Erzeugnissen. Ökologische Methoden hingegen gehen häufig einher mit einer vergleichsweise deutlich geringeren Produktion. ${ }^{50}$ Als eine Möglichkeit, das Überangebot zu reduzieren, wurde daher der ökologische Landbau als agrarpolitische Alternative nun erstmals auch von staatlicher Seite politisch und finanziell gefördert. 1983 erhielt das Österreichische Lebensmittelgesetz den Zusatz "Landwirtschaftliche Produkte aus biologischem Landbau und daraus hergestellte Folgeprodukte« als erstes Regelwerk für ökologische Produktionsmethoden. ${ }^{51}$ Die Erhaltung einer vielfältigen Landschaft war als Nebenprodukt der ökologischen Bewirtschaftung besonders für die wachsende Tourismusbranche der Steiermark von Bedeutung.

Seit Ende der 1970er Jahre zählten umweltbewusste Konsument* innen zur wachsenden Zahl der Abnehmer*innen von Öko-Produkten. Erstmals überstieg die Nachfrage das Angebot. Verkauft wurden ökologisch produzierte Waren größtenteils ab Hof, ohne besondere Kennzeichnung. Audrey Vankeerberghen stellt für Belgien fest, was auch für die Situation in Österreich galt: »At that time, there were two ways for buyers to identify organic goods: personal relationships of trust between producers and consumers, or private organic standards. ${ }^{52}$ Anfang der 1980er entstanden in Österreich

49 Vgl. European Commission 2019, S. 22.

50 Vgl. European Commission 2019, S. 6. Siehe dazu auch Einleitung zu diesem Band.

51 Vgl. Jurtschitsch 2010; BMCF 2017. Ab dem EWR-Beitritt Österreichs 1994 traten die EU-Richtlinien für ökologischen Landbau in Kraft und lösten damit frühere Regelungen $a b$.

52 Vankeerberghen 2012, S. 104. 
erste ökologische Verbraucher-Erzeuger Initiativen und Bio-Läden. »Der inhaltliche Schwerpunkt« schreibt Vogt über die Tendenzen im ökologischen Landbau »verschob sich vom Erhalt einer bäuerlichen Lebenswelt zur Entwicklung einer umweltschonenden, dauerfähigen Landbau- und Lebensweise «. ${ }^{53}$

1979 spaltete sich von der »Förderungsgemeinschaft für gesundes Bauerntum« der als reiner Anbauverband geplante »Bundesverband organisch-biologisch wirtschaftender Bauern Österreich« ab (später »Ernte für das Leben«). Dessen Mitglieder waren deutlicher an neuen Absatzmöglichkeiten, Vermarktungsstrategien und besseren Preisen interessiert. Die Mitgliedschaft war auch Voraussetzung für die Verwendung eines eigenen Siegels als Auszeichnung für eine ökologische Produktion. Der Verein wurde rasch zum mitgliederstärksten Verband Österreichs. Die Zentralisierungs- und Institutionalisierungsbestrebungen (und damit einhergehend die Etablierung von einheitlichen Produktionsstandards) kulminierten in der Gründung des zentralen Dachverbandes Bio Austria. ${ }^{54}$ Vankeerberghen erkennt dahinter eine EU-weite Entwicklung: »Originally a matter of diverse specification, often informal and based on trust, it became a matter of concern for the different organisations and associations within the organic movement, and then became a matter of legislation, enforced by commercial inspection companies authorised by individual European countries «. ${ }^{55}$

Mit dem EU Beitritt Österreichs 1995 sanken die Preise für landwirtschaftliche Produkte im erweiterten Markt. Mit Spezialprodukten wie Kernöl, Wein und Öko-Waren sollten den Produzent*innen einträgliche Nischen eröffnet werden. ${ }^{56}$ Gleichzeitig wurden LebensmittelHandelsketten als Abnehmer für Öko-Waren gewonnen. Für viele Produzent*innen tat sich damit ein lukrativer Markt auf, jedoch mussten Öko-Waren nun auch »regalfähig« werden. Kritiker*innen bemängelten, dass nun auch im Öko-Bereich die Lebensmittelqualität

\footnotetext{
53 Vogt 2001b, S. 49.

54 Vgl. Schermer 2005.

55 Vankeerberghen 2012, S. 105.

56 Vgl. Karner 2000; Sassatelli und Scott 2001; Schermer 2015.
} 
zugunsten von Quantität und Aussehen an Bedeutung verlor. Der Öko-Landbau hatte die Blase der Direktvermarktung und ErzeugerVerbraucher Verbände verlassen, „Wachsen oder Weichen« galt nun auch für den ökologischen Landbau, womit gleichzeitig die Konventionalisierungsdebatte einsetzte. ${ }^{57}$ Ploeg hält dazu fest:

»Started as an alternative praxis developed from a variety of sources that all differed from, and strongly criticized conventional farming, the techniques and logos have now been taken over by large-scale entrepreneurial and corporate farms (that consider organic agriculture to be an interesting economic opportunity, no more and no less). It has, in short, largely been conventionalized. ${ }^{58}$

Herr Danninger, der seit den 1990ern ökologische Tafeläpfel an den Lebensmittelhandel liefert (und der in Ploegs Begrifflichkeiten als entrepreneurial farmer gelten kann), kommentiert diese Entwicklung im Interview. Dabei bemerkt er, dass die neuen Konsumansprüche längst nicht mehr nur im Supermarkt von Bedeutung sind:

»Also damals [vor der Konventionalisierung, Anm. EK] haben wir unsere Äpfel auch verkauft, weil die Leute Bio-Äpfel haben wollten, die nach was schmecken. Und jetzt ist es nicht einmal im Bio-Laden mehr wirklich leicht, Äpfel mit Flecken zu verkaufen.« (Interview am 9.8.2018)

Staatliche Förderungen und neue Absatzmöglichkeiten motivierten viele Landwirt*innen seit Anfang der 1990er zum Umstieg auf ökologischen Landbau. Dominierte bis dahin peasant farming unter den ÖkoBetrieben, begann nun die Zeit des entrepreneurial farming auch im ökologischen Landbau - als den Bedürfnissen des Weltmarktes angepasste Rohstofflieferanten. Ploeg hält fest: „Entrepreneurial farmers are highly market-dependent (both for inputs and outputs), which is in stark contrast to peasants who generally try to distantiate their farming practices from markets through a multitude of often ingenious mechanisms «. ${ }^{59}$

57 Vgl. Groier 2013; Guthman 2004, 2014; Vankeerberghen 2012.

58 Ploeg 2018b, S. 264.

59 Ploeg 2018b, S. 2. 
Für viele der neuen Öko-Entrepreneure war die Erfahrung jedoch keineswegs nur positiv. Schon Ende der 1990er stellte eine Vielzahl von ihnen die ökologische Produktion wieder ein, aus unterschiedlichen Gründen: hohe Investitionskosten, der hohe und gleichzeitig wenig lohnende bürokratische Aufwand für Biokontrollen und Förderungen, Ertragseinbußen aufgrund der Umstellung und der erhöhte Arbeitsaufwand. $^{60}$

Die Entwicklung hin $\mathrm{zu}$ einer eco-entrepreneurial agriculture, in Anlehnung an Ploegs Begriff, blieb dabei immer partiell. Bis heute ziehen es zahlreiche Produzent*innen vor, peasant farmers zu bleiben, beziehungsweise zu werden - ein Phänomen, dass sich weder auf bestimmte globale Regionen noch auf den ökologischen Landbau beschränkt. ${ }^{61}$ Die Fallbeispiele unserer Studie zeigen dabei auf, dass es gerade auch die affektiven Beziehungen zu Bodenlebewesen, Tieren und Menschen sind, die mit einer produktivistischen Denkweise unvereinbar bleiben. Herr Adam, der bewusst viel händisch arbeitet und auf Vielfalt und samenfeste Sorten auf seinem Gemüsehof wert legt, stellt selbstkritisch fest:

»Das ist nicht wirtschaftlich. Und das ist mir schon bewusst in Wirklichkeit, aber die Wirtschaft ist für mich nicht der Punkt. Mein Punkt ist, meinen Boden zu verbessern und eine gute Ernährung zu kreieren. Und eine gute Ernährung ist für mich ein bisschen gleichwertig mit Medizin. « (Interview am 20.08.2017)

Das Zitat spiegelt Ansätze der Ernährungsreform wieder, die Lebensmittel als Heilmittel betrachtete. ${ }^{62}$ Am Hof von Familie Adam werden Boden und Pflanzen nicht gezwungen, an dem Ort oder zu dem Zeitpunkt zu wachsen, an dem es für die Betriebsleitung (oder den Weltmarkt) am profitabelsten ist. Frau Adam ergänzt: »Was uns wichtig ist, so wenig Bodenbewegung wie möglich machen, oder? Dem Boden die Möglichkeit

60 Vgl. Freyer u.a. 2001; Groier 2005.

61 Vgl. Ploeg 2018b.

62 Vgl. Baumgartner 1998. 
zu lassen, sich zu entwickeln und sich so anzulegen, wie es für ihn gesund ist und gut ist (Interview am 20.08.2017).

Im Sinne von Puig de la Bellacasas human-soil communities ist hier der Mensch nicht mehr nur Abnehmer und Nutznießer der Leistungen von Boden und Bodenlebewesen, sondern Teil einer Gemeinschaft. »In other words, within these conceptions, to properly care for the soil humans cannot be only producers or consumers in the community of soil making organisms but must work, and be, in relation to soil as a significant living world «. ${ }^{63}$ Der Boden, beziehungsweise die Bodenlebewesen sind eigenständige Akteure die beobachtet werden, um mit ihnen zusammen $\mathrm{zu}$ arbeiten und $\mathrm{zu}$ leben, um sich gegenseitig $\mathrm{zu}$ versorgen. Ploegs Begriff von co-production deutet in dieselbe Richtung:

»From the point of view of co-production, the resource base of the farm can be conceptualized as composed by living nature and the capacity to deal with it. Living nature is embodied in the land (and the soil biology it contains), in crops, water, animals, and the local ecosystem. Together with this comes the capacity of farmers to understand, deal with, develop and convert living nature into food. ${ }^{64}$

Co-production ist für Ploeg ein Charakteristikum des peasant farming:»By contrast, entrepreneurial and corporate agriculture tend to minimize the role of living nature because, within these structurally different contexts, it represents a nuisance and a limit. « ${ }^{65}$

Für die Viehwirtin Frau Conrad steht in ihren Erzählungen weniger die Beziehung zu Bodenlebewesen und Pflanzen im Vordergrund, sondern ihre Beziehung zu den Tieren:

»Man schreibt ja schon vor, die Tiere sind zu alt, sie gehören weg. Ich finde, wenn ich der Meinung bin, mein Ziegenbock ist mit acht Jahren nicht zu alt, ist es meine Entscheidung, ich möchte das nicht vorgeschrieben bekommen. Ich möchte eine Wertschätzung für das Tier, das mir ein Leben lang gedient

63 Puig de la Bellacasa 2015, S. 706, Hervorhebung original.

64 Ploeg 2018b, S. 20, Hervorhebung original.

65 Ploeg 2018b, S. 20, Hervorhebung original; siehe auch Ploeg 2010. 
hat, das finde ich auch wichtig, es muss nicht immer alles nur auf Gewinn aufgebaut sein.« (Interview am 05.07.2018)

Frau Conrads Sorge um das Wohl ihrer Tiere führte zu der paradoxen Situation, dass sie von den zuständigen Behörden eine Anzeige wegen falscher Tierhaltung erhielt, als sie sich weigerte, ein Kälbchen nach der Geburt sofort von seiner Mutter zu trennen. Puig de la Bellacasas Feststellung zu human-soil-relations als care-Arbeit scheint hier, erweitert auf more-than-human-relations, besonders zutreffend:

"Valuing care by refficiency< standards transforms its practice into a managed >conduct < to be monitored (Latimer, 2000). That is why, in contexts of managerial control that underestimates care's value and even penalize its practice, acts of care can be considered as a kind of resistance. " $" 66$

Gleichzeitig, und weitab von jeder Agrarromantik, sind sich die Landwirt*innen in unserer Studie immer bewusst, dass ihr Hof ein Einkommen erwirtschaften muss. In ihren Praktiken der Produktion, Verarbeitung und Vermarktung suchen sie allerdings nach Möglichkeiten, dies ohne Ausbeutung von Bodenlebewesen, Tieren oder Menschen, einschließlich ihrer eigenen Arbeitskraft, $\mathrm{zu}$ erreichen (siehe Kapitel Heistinger).

Seit den Anfängen ökologischer Landbaumethoden wird von Praktiker*innen der Wunsch nach Wertschätzung für diese Form der zeitund arbeitsintensiven Produktion geäußert, sowie eine faire Entlohnung für landwirtschaftliche care-Arbeit gefordert. ${ }^{67}$ Von staatlicher Seite wird diese care-Arbeit einer sorgsamen Landwirtschaft in Förderungen wie Flächenprämien für umweltschonend(er)e landwirtschaftliche Praktiken umgerechnet, als bürokratisch geregelte, standardisierte und überprüfbare Formen der Entlohnung - „valuing care by refficiency< standards«. Gabriele Sorgo stellt in ihrem Kapitel dar, wie unentlohnte, reproduktive (oft als unproduktiv deklarierte) care-Arbeit in einer marktorientierten Gesellschaft strukturell entwertet wird. Gleich-

66 Puig de la Bellacasa 2015, S. 708.

67 Vgl. Jurtschitsch 2010; Vogt 2000. 
zeitig baut die Gesellschaft weiterhin darauf auf, dass care-Arbeiten als kostenfreie »Liebesdienste« dauerhaft zur Verfügung stehen. ${ }^{68}$ Im Sinne einer human-soil-community drückt sich dieser Gedanke nicht nur in entwerteter Arbeit des Menschen, sondern auch des Bodens und anderer Lebewesen aus. So kommentiert Frau Adam: "Wir sind ganz gut im Kalkulieren. Aber dann gibt es trotzdem so Spitzenzeiten und dann haben sich die Pflanzen bemüht, wir haben uns bemüht und dann nimmt es uns keiner ab« (Interview am 20.08.2017).). Der Einsatz der Hofgemeinschaft, die über die menschliche Arbeitskraft hinausgeht, bleibt unentlohnt.

\section{Schlussbemerkung}

Die Aufgabe dieses Kapitels war es, die historischen Entwicklungen im ökologischen Landbau für die Forschungsregion Oststeiermark nachzuzeichnen, um die Fallbeispiele der vorliegenden Studie zu kontextualisieren. Dabei zeigt sich, dass die ökologische Landwirtschaft ein heterogenes Feld an Akteur*innen, Motivationen und Praktiken ist, das sich nicht schlicht auf die Position einer (agrarromantischen) Kritik an der industriellen, chemisch-technischen Landwirtschaft reduzieren lässt. Im Besonderen wurden Care Diskurse und Praktiken thematisiert, wie sie in unterschiedlichen Bezügen in der Geschichte des ökologischen Landbaus sichtbar werden. Die Erweiterung feministischer Care Diskurse durch Puig de la Bellacasas Konzepte von soil care erweist sich als besonders hilfreich, um Praxen und Vorstellungen eines Zusammenlebens von menschlichen und anderen Akteur*innen, soil-communities, als Versorgungsgemeinschaft $\mathrm{zu}$ betrachten. Divergierende Bedürfnisse werden wahrgenommen und dennoch, oder gerade dadurch, Grundnahrungsmittel abseits von Ausbeutungsverhältnissen (von der Ausbeutung des Bodens bis zur Selbstausbeutung) produziert.

68 Ausgehend von feministischen Diskursen seit den 1970ern wird faire Entlohnung für Versorgungsarbeit seit der Jahrtausendwende wieder verstärkt in den Sozial- und Kulturwissenschaften thematisiert; siehe etwa Binder u.a. 2019. 